\title{
Identification of the involvement of LOXL4 in generation of keratocystic odontogenic tumors by RNA-Seq analysis
}

\author{
Wei-Peng Jiang ${ }^{1}$, Zi-Han Sima ${ }^{1}$, Hai-Cheng Wang ${ }^{1}$, Jian-Yun Zhang ${ }^{1}$, Li-Sha Sun ${ }^{2}$, Feng Chen ${ }^{2}$ and Tie-Jun Li ${ }^{1}$ \\ Keratocystic odontogenic tumors (KCOT) are benign, locally aggressive intraosseous tumors of odontogenic origin. KCOT have a higher \\ stromal microvessel density (MVD) than dentigerous cysts (DC) and normal oral mucosa. To identify genes in the stroma of KCOT \\ involved in tumor development and progression, RNA sequencing (RNA-Seq) was performed using samples from KCOT and primary \\ stromal fibroblasts isolated from gingival tissues. Seven candidate genes that possess a function potentially related to KCOT \\ progression were selected and their expression levels were confirmed by quantitative PCR, immunohistochemistry and enzyme-linked \\ immunosorbent assay. Expression of lysyl oxidase-like 4 (LOXL4), the only candidate gene that encodes a secreted protein, was \\ enhanced at both the mRNA and protein levels in KCOT stromal tissues and primary KCOT stromal fibroblasts compared to control \\ tissues and primary fibroblasts $(P<0.05)$. In vitro, high expression of LOXL4 could enhance proliferation and migration of the human \\ umbilical vein endothelial cells (HUVECs). There was a significant, positive correlation between LOXL4 protein expression and MVD in \\ stroma of KCOT and control tissues $(r=0.882$ ). These data suggest that abnormal expression of LOXL4 of KCOT may enhance \\ angiogenesis in KCOT, which may help to promote the locally aggressive biological behavior of KCOT.
} International Journal of Oral Science (2013) 6, 31-38; doi:10.1038/ijos.2013.96; published 20 December 2013

Keywords: angiogenesis; keratocystic odontogenic tumor; lysyl oxidase-like 4; RNA-sequencing; tumor stromal fibroblast

\section{INTRODUCTION}

Keratocystic odontogenic tumors (KCOT) are benign, intraosseous tumors of odontogenic origin, with a characteristic lining of parakeratinized, stratified squamous epithelium and locally aggressive behavior. ${ }^{1}$ In some patients, KCOT are associated with nevoid basal cell carcinoma syndrome. ${ }^{2}$ The traditional designation for KCOT has been as an odontogenic keratocyst, which emphasizes the cystic nature of these lesions. ${ }^{3}$ Previous studies demonstrated that PTCH1, a tumorsuppressor gene mapping to chromosome 9q22.3-q31, is involved in KCOT etiology. ${ }^{4}$ However, since germline mutations in PTCH1 are less frequent in patients with sporadic KCOT than in those with nevoid basal cell carcinoma syndrome-related KCOT, the pathogenesis of KCOT remains poorly characterized.

The stroma of KCOT has been reported to promote tumor progression. ${ }^{5}$ Fibroblasts are the major component of stromal cells and secrete various cytokines, chemokines and extracellular matrix proteins to form the microenvironment that stimulates epithelial cell growth. ${ }^{6-7}$ Several notable differences exist between KCOT stroma and control tissues. For example, the microvessel density (MVD) in KCOT stroma is greater than that in control tissues from dentigerous cysts (DC) or normal oral mucosa. ${ }^{8}$ The stromal collagen fibers of KCOT also differ from those found in DC, but are similar to those seen in unicystic ameloblastoma. $^{9-10}$ Furthermore, greater osteoclastogenic capacity was detected in KCOT fibroblasts cocultured with Raw264.7 cells. ${ }^{11}$ These findings support the hypothesis that the stroma of KCOT should be recognized not only as structural support of the cyst wall, but also as a contributory factor to the neoplastic behavior of the cyst.

The advent of massive parallel sequencing technology has provided a powerful tool to study transcriptome changes through RNA sequencing (RNA-Seq). ${ }^{12}$ By sequencing the entire transcriptome of tumors and matched control tissues, their expression levels can be compared. ${ }^{13-14}$ RNA-Seq has a wider range of detection and higher sensitivity than microarrays and does not require advance knowledge of sequence information. In this study, we used RNA-Seq to explore the role of the stroma in KCOT pathogenesis and the progression of angiogenesis.

\section{MATERIALS AND METHODS}

Patient samples

We selected two fresh tissue specimens of mandibular adult-type KCOT from a female, aged 19 years, and a male, aged 35 years, index patient based on pathological diagnosis and clinical features. We also collected two excess healthy gingival tissue (GG) specimens from a female, aged 22 years, and a male, aged 29 years, after extraction of the third molar (wisdom tooth). No clinical signs of inflammation or bone destruction were evident in these healthy volunteers. Stromal fibroblasts were isolated and cultured as described previously. ${ }^{11}$ All

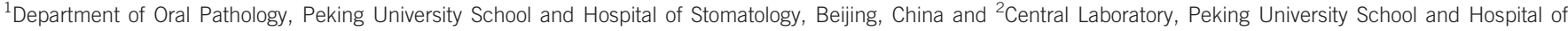
Stomatology, Beijing, China

Correspondence: Dr TJ Li, Department of Oral Pathology, Peking University School and Hospital of Stomatology, 22 South Zhongguancun Avenue, Beijing 100081, China

E-mail: litiejun22@vip.sina.com

Dr F Chen, Central Laboratory, Peking University School and Hospital of Stomatology, 22 South Zhongguancun Avenue, Beijing 100081, China

E-mail: moleculecf@gmail.com

Accepted 12 November 2013 
samples were acquired at Peking University, Hospital of Stomatology, following institutional review board approval of their use for RNA-Seq analysis.

\section{Preparation of cDNA and sequencing}

Preparation of cDNA and RNA-Seq was performed as shown in Supplementary Figure S1.

\section{Alignment statistics}

Clean reads from the KCOT and control samples were mapped to the reference genome and gene sequences using SOAP2 - a significantly improved version of the short oligonucleotide alignment program that both reduces computer memory usage and increases alignment speed at an unprecedented rate. ${ }^{15}$ Mismatches no larger than $m$ bases (default $m$ value $=5$ ) were allowed in the alignment. ${ }^{16}$ Allowing a smaller number of two or three mismatches did not affect the analysis (data not shown). Only reads that mapped uniquely were included in the analysis, unless indicated otherwise.

\section{Candidate gene filtering and mRNA expression validation} We filtered the data for genes expressed at abnormal levels in the stroma of KCOT compared to controls ( $F$ values $\leqslant 0.001$ ), as described previously, ${ }^{17}$ to identify those that may play a role in the pathogenic levels of angiogenesis observed in KCOT patients.

The mRNA levels of candidate genes were quantified using quantitative PCR (QPCR). QPCR was performed on a 7500 Fast System (Applied Biosystems, Foster City, CA, USA) according to a previously described protocol. ${ }^{18}$ All primers were purchased from Invitrogen (Carlsbad, CA, USA) and are summarized in Supplementary Table S1.

\section{Immunohistochemistry}

Twenty formalin-fixed and paraffin-embedded KCOT and DC tumors were obtained from primary surgeries in the Department of Pathology, Peking University School and Hospital of Stomatology. Ten normal GG tissues from wisdom teeth extractions were also collected to serve as controls.

The protocol for lysyl oxidase-like (LOXL4) and CD105 immunohistochemical analysis was based on Weise and Gadbail. ${ }^{8-19}$ Polyclonal rabbit anti-human LOXL4 (1:2000; ab88186; Abcam, Cambridge, UK) and CD105 (1:30; ZM-0297; Beijing Zhongshan, Beijing, China) were used as the primary antibodies. Each section was accompanied by positive and negative controls. Positive controls consisted of human pancreas for LOXL4 and human tonsil for CD105. Negative controls were obtained by omission of the primary antibody and replacement with non-immune serum. The slides were independently reviewed by two observers (TJL and FC) to confirm the analysis.

We randomly selected six fields at $\times 100$ magnification, and counted cells at $\times 400$ magnification. Cells with cytoplasmic staining with diaminobenzidine were considered positive for LOXL4. CD105 analysis was carried out using an Olympus BX51 research microscope (Olympus, Tokyo, Japan). CD105-positive vascular endothelial cells were clearly identified by brown cytoplasmic staining. Distinct countable microvessels were assessed using the criteria described by Jaeger. ${ }^{20}$ MVD of the adjacent connective tissue was measured using the method of Gadbail. ${ }^{8}$

\section{Enzyme-linked immunosorbent assay}

Due to the availability of material, LOXL4 levels were measured by enzyme-linked immunosorbent assay (ELISA) in both primary fibroblast cell supernatants and cell lysates. A commercially available ELISA kit (E95554Hu; Uscn, Houston, TX, USA) for LOXL4 was used according to the manufacturer's instructions. The plates were read at an absorbance of $450 \mathrm{~nm}$ using a Spectra Max Plus plate reader (ELx808; BioTek, Winooski, VT, USA). Results were normalized to the number of tested cells by measuring the protein concentration of each sample before the ELISA using the Pierce BCA Protein Assay Kit (Thermo, Boston, MA, USA). Measurements were performed in triplicate.

\section{Construction of expression plasmid LOXL4}

Human LOXL4 cDNA was purchased from Sino Biological and cloned in pCMV/hygro (CV004; Sino Biological, Beijng, China) for transient overexpression with a DNA Ligation Kit (2011A; TAKARA, Kyoto, Japan).

\section{Western blot analysis}

Total protein lysates from human umbilical vein endothelial cells (HUVECs) were resolved on $8 \%$ sodium dodecyl sulfate-polyacrylamide gel eletrophoresis (SDS-PAGE), then transferred to nitrocellulose membrane or polyvinylidene fluoride membrane, and underwent immunoblotting with LOXL4 antibody (1:2000; ab88186; Abcam, Cambridge, UK). The secondary antibodies were goat anti-rabbit at 1:5000 (1:2000; sc2004; Santa Cruz Biotechnology, Dallas, TX, USA).

\section{Endothelial cell proliferation assay}

HUVECs $\left(1 \times 10^{4}\right.$ cells per well) were cultured for $24 \mathrm{~h}$ in 96 -well tissue culture plates (Corning, New York, NY, USA) in Dulbecco's modified Eagle's medium (DMEM) supplemented with 10\% fetal bovine serum (FBS) and $0.1 \mathrm{mg} \cdot \mathrm{mL}^{-1}$ heparin. Cell proliferation was measured by Cell Counting Kit-8 (Dojindo, Tokyo, Japan).

\section{Endothelial cell transmigration assay}

For the scratching assay, HUVECs $\left(8 \times 10^{5}\right.$ cells in $3 \mathrm{~mL}$ DMEM) were seeded in $60-\mathrm{mm}$ diameter culture dishes. The medium was changed to serum-free OPTI.MEM the next morning for synchronization. Four hours later, scratching was made and fresh medium without FBS was added. Five fields were randomly selected in each dish for recording gap distances immediately following scratching and $6 \mathrm{~h}$ afterwards.

For Boyden chamber assay, $200 \mu \mathrm{L}$ of HUVECs suspension $\left(2 \times 10^{4} \mathrm{~mL}^{-1}\right.$ in DMEM without FBS $)$ was placed in the upper chamber, and $600 \mu \mathrm{L}$ DMEM with $10 \%$ FBS was placed in the lower chamber. After incubation for $4 \mathrm{~h}$, the cells on the upper surface were removed, and the cells on the underside were fixed and stained. Each treatment was performed in triplicate wells, and the mean number of cells in each well was counted from four randomly chosen fields under light microscopy $(\times 200)$.

\section{Statistical analysis}

Data are the means of three individual experiments performed in triplicate and analyzed using one-way analysis of variance, a Student's $t$-test for independent samples, a Bonferroni test for multiple comparisons and a Pearson's rank correlation test. $P$ values $<0.05$ were considered to indicate statistical significance.

\section{RESULTS}

\section{Sequence enrichment}

The RNA-Seq reads were first aligned based on a method described previously. ${ }^{21}$ The majority of reads from each sample $(77.36 \%-84.88 \%)$ aligned successfully to the human genome (Table 1). The sequences that 
Table 1 Summary of sequence enrichment during RNA-Seq of keratocystic odontogenic tumors and normal gingival tissues

\begin{tabular}{|c|c|c|c|c|}
\hline \multirow[b]{2}{*}{ Map to genome/gene } & \multicolumn{2}{|c|}{ Map togenome } & \multicolumn{2}{|c|}{ Map to gene } \\
\hline & Reads number & Percentage/\% & Reads number & Percentage/\% \\
\hline \multicolumn{5}{|l|}{ ксот } \\
\hline Total basepairs & 4715007840 & 100.00 & 4715007840 & 100.00 \\
\hline Total mapped reads & 44465709 & 84.88 & 40525636 & 77.36 \\
\hline Perfect match & 33038565 & 63.06 & 40525636 & 62.69 \\
\hline$\leqslant 5 \mathrm{bp}$ mismatch & 11427144 & 21.81 & 7685059 & 14.67 \\
\hline Multiposition match & 2632667 & 5.03 & 15211223 & 29.04 \\
\hline Total unmapped reads & 7923267 & 15.12 & 11863340 & 22.64 \\
\hline \multicolumn{5}{|l|}{ GG } \\
\hline Total reads & 49781578 & 100.00 & 49781578 & 100.00 \\
\hline Total basepairs & 4480342020 & 100.00 & 4480342020 & 100.00 \\
\hline Total mapped reads & 41916300 & 84.20 & 38531845 & 77.40 \\
\hline
\end{tabular}

GG, gingival primary stromal fibroblast cells; KCOT, keratocystic odontogenic tumors; RNA-seq, RNA-sequencing.

Table 2 Genes identified as up-regulated or down-regulated by RNA-Seq and microarray analysis

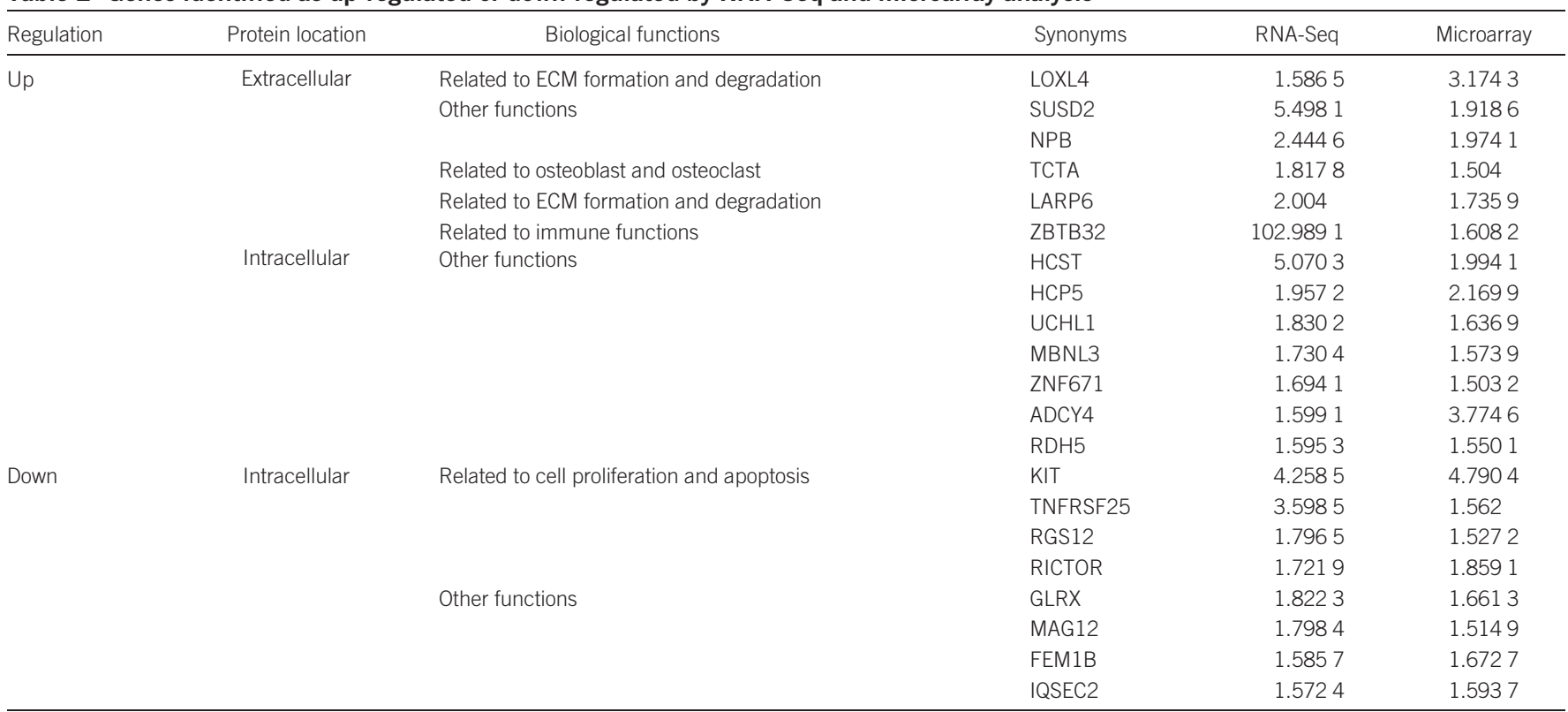

ECM, extracellular matrix; LARP6, La ribonucleoprotein domain family, member 6; LOXL4, lysyl oxidase-like 4; RNA-Seq, RNA sequencing; TCTA, T-cell leukemia translocation-associated gene.

did not align are likely to be polyclonal, of low quality, or have origins outside the reference human genome.

\section{RNA-Seq analysis of gene expression in KCOT, DC and normal gingival tissues}

We compared the RNA-Seq data to microarray results from our laboratory (data not shown), which compared the mRNA levels in six primary KCOT stomal fibroblasts to those in six DC. Genes up- or downregulated in both the RNA-Seq and microarray data were selected for further analysis (Table 2). We selected candidate genes with a function potentially related to KCOT generation (Figure 1 and Table 3). As secreted proteins may affect adjacent tumor epithelial cells, we focused on LOXL4, a copper-containing amine oxidase, which belongs to the LOX family, as it was the only one of the seven candidate genes that encoded a secreted protein.

\section{Verification of RNA-Seq results}

QPCR analysis was performed to confirm the RNA-Seq and microarray results by verifying mRNA expression levels of the seven candidate genes (Table 3). The expression levels of LOXL4, T-cell leukemia translocation-associated gene (TCTA), La ribonucleoprotein domain family, member 6 (LARP6) and regulator of G-protein signaling 12 (RGS12) in three samples of KCOT primary stromal fibroblasts, GG stromal primary fibroblasts and DC stromal primary fibroblasts were consistent with the RNA-Seq and microarray data ( $P<0.05$; Figure $2 \mathrm{a})$. There were no significant differences among the expression levels of 


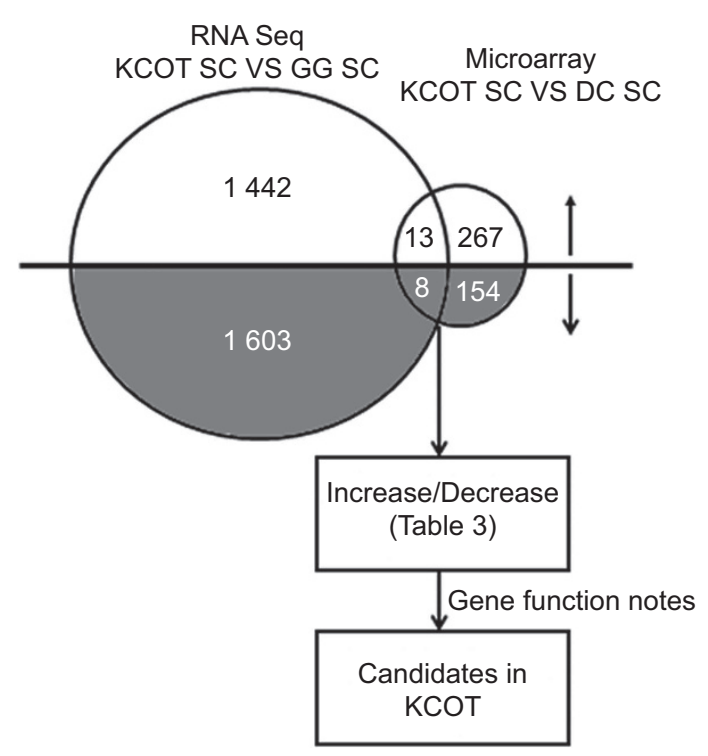

Figure 1 Search outline for candidate genes identifed by RNA-Seq. Genes differentially expressed in KCOT, primary SC and healthy GG were first identified by RNA-Seq. The RNA-Seq results were compared to microarray data, which was performed to identify differentially expressed genes in KCOT primary stromal fibroblasts and in DC primary SC (data not shown). The genes were then subjected to further selection based on their function, to identify candidate genes that may affect the progression of KCOT. DC, dentigerous cysts; GG, gingival primary stromal fibroblast cells; KCOT, keratocystic odontogenic tumors; RNA-Seq, RNA sequencing; SC, stromal fibroblast cell.

KIT, TNFRSF25 and RICTOR in stromal primary fibroblasts $(P>0.05$; data not shown). To confirm the expression of the candidate gene LOXL4, we quantified the expression of LOXL4 mRNA in three groups: five healthy GG samples, three DC samples and five KCOT samples. All stromal fibroblasts were derived from freshly isolated tissues. The expression of LOXL4 mRNA was significantly higher in the KCOT tissues than in the DC (3.7-fold) and GG (2.3-fold) tissues $(P<0.05$; Figure $2 \mathrm{~b}$ ), consistent with the RNA-Seq and microarray results.

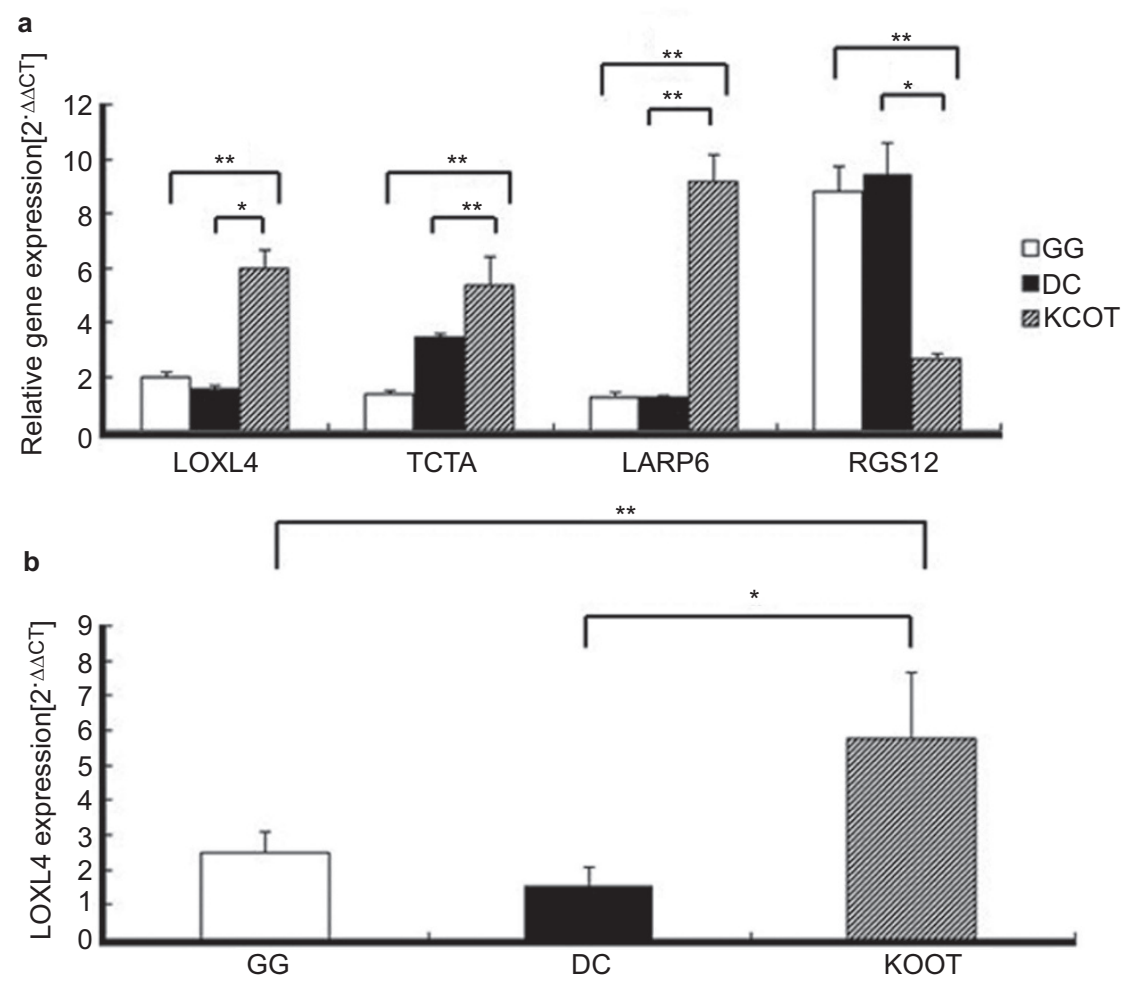

Figure 2 Confirmation of RNA-Seq results. (a) QPCR analysis of TCTA, LARP6, RGS12 and LOXL4 mRNA expression in primary stromal fibroblasts of KCOT, DC and healthy GG. The data were consistent with the RNA-Seq and microarray results. (b) QPCR analysis of LOXL4 mRNA expression in primary stromal fibroblasts of KCOT, DC and GG. Each bar corresponds to the mean \pm s.d. of three independent experiments performed in triplicate with three technical repeats. $* P<0.05, * * P<0.001$. DC, dentigerous cysts; GG, gingival primary stromal fibroblast cells; KCOT, keratocystic odontogenic tumors; LARP6, La ribonucleoprotein domain family, member 6; LOXL4, Iysyl oxidase-like 4; QPCR, quantitative PCR; RGS12, regulator of G-protein signaling 12; RNA-Seq, RNA sequencing; TCTA, T-cell leukemia translocation-associated gene. 
Table 3 Candidate genes possibly involved in keratocystic odontogenic tumor progression

\begin{tabular}{lllll}
\hline Name & RNA-Seq & Microarray & Regulation & \\
\hline LARP6 & 2.004039 & 1.7358851 & Up & Regulates the coordinated translation of type I collagen alpha-1 and alpha-2 mRNAs, C01A1 and CO1A2 \\
LOXL4 & 1.856549 & 3.1743474 & Up & Modulate the formation of a collagenous extracellular matrix \\
TCTA & 1.8177639 & 1.5039967 & Up & Required for cellular fusion during osteoclastogenesis \\
KIT & 4.25854 & 4.790408 & Down & This is the receptor for stem cell factor (mast cell growth factor) \\
TNFRSF25 & 3.598541 & 1.561969 & Down & The protein encoded by this gene is a member of the TNF-receptor superfamily \\
RGS12 & 1.796471 & 1.527165 & Down & This protein may play a role in tumorigenesis \\
RICTOR & 1.721887 & 1.85907 & Down & Subunit of mTORC2, which regulates cell growth and and survival in response to hormonal signals \\
\hline
\end{tabular}

ECM, extracellular matrix; LARP6, La ribonucleoprotein domain family, member 6; LOXL4, lysyl oxidase-like 4; RGS12, regulator of G-protein signaling 12; TCTA, T-cell leukemia translocation-associated gene; TNF, tumor-necrosis factor.

a

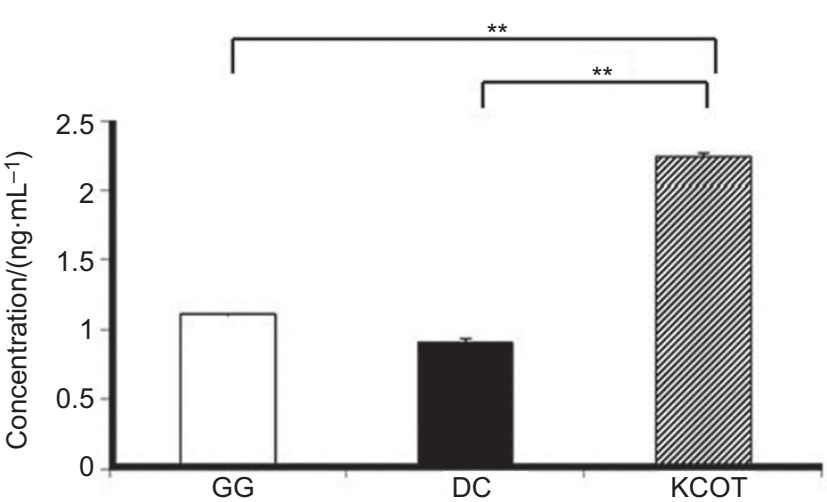

LOXL4 protein expression level in cell lysates

b

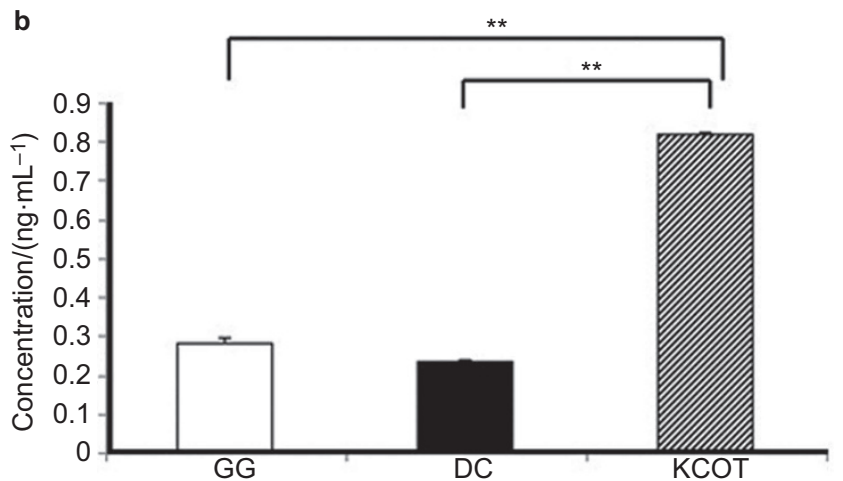

Figure 3 LOXL4 is overexpressed in the stroma of KCOT. (a) LOXL4 concentration in the supernatants of primary fibroblasts derived from KCOT, GG and DC ( $n=3$ per tissue) by ELISA. (b) LOXL4 concentration in the cell lysates of primary fibroblasts derived from KCOT, GG and DC ( $n=3$ per tissue) by ELISA. Data are the means of triplicates from a representative experiment \pm s.d.; $* P<0.05$, $* * P<0.001$. DC, dentigerous cysts; ELISA, enzyme-linked immunosorbent assay; GG, gingival primary stromal fibroblast cells; KCOT, keratocystic odontogenic tumors; LOXL4, lysyl oxidase-like 4.

\section{LOXL4 protein expression is upregulated in KCOT fibroblasts}

LOXL4 protein levels were quantified in the supernatants and lysates of nine primary stromal fibroblast cell lines (three KCOT, three DC and three normal GG tissues) by ELISA. LOXL4 was detected in all nine fibroblast cell line supernatants and lysates (Figure $3 \mathrm{a}$ and $3 \mathrm{~b}$ ). The LOXL4 protein level was significantly higher in the supernatants and lysates of KCOT stromal fibroblasts than in stromal fibroblasts derived from DC or GG tissues. Compared to KCOT stromal fibroblasts, the levels of LOXL4 were 2.47- and 2.04-fold lower in DC and GG stromal fibroblast supernatants, respectively, and 3.46- and 2.87-fold lower in DC and GG stromal fibroblast lysates, respectively (Figure 3b; $P<0.001$ ).

\section{Immunohistochemical analysis of LOXL4}

Immunohistochemical analysis was performed to quantify LOXL4 protein expression in KCOT, DC and GG tissue samples. Few fibroblasts in DC and GG tissues expressed LOXL4, and the immunoreactivity was faint or weak (Figure 4a1, 4a2, 4a4 and 4a5). However, intense LOXL4 immunostaining was observed in the cytoplasm of stromal fibroblasts in KCOT tissues (Figure 4a7 and 4a8). We also identified significant differences in the numbers of cells stained by LOXL4 and the MVD of the KCOT, DC and GG samples $(P<0.05)$.

Previous studies have confirmed that the LOX family members LOX and LOXL2 can enhance angiogenesis in vivo and in vitro. ${ }^{22-23}$ As a member of the LOX family, LOXL4 has a similar structure to LOX and LOXL2, so we hypothesized that it may also be related to angiogenesis. We used CD105, a marker of angiogenesis, to quantify angiogenesis in the stroma of KCOT, DC and GG tissues. CD105 was expressed in $100 \%$ of KCOT stromal endothelial cells, $45.73 \%$ of DC stromal endothelial cells and 52.68\% of GG stromal endothelial cells. Similar to LOXL4, the quantity of CD105-positive vessels was higher in the stroma of the KCOT samples than the DC and GG tissues (Figure 4a3, 4a6 and 4a9).

Comparison of the three groups by one-way analysis of variance revealed a statistically significant difference $(P<0.001)$. Bonferroni analysis did not indicate a significant difference in LOXL4 and MVD between the DC and GG samples $(P=0.358)$. However, the differences between KCOT samples and DC $(P<0.001)$ and GG $(P<0.001)$ samples were statistically significant. Significant positive correlations were observed between the LOXL4 and MVD in all three groups (correlation coefficient $(r)=0.882 ; P<0.001$; Figure 4 a and Table 4$)$.

Table 4 Microvessel density and LOXL4 in keratocystic odontogenic tumors, dentigerous cysts and gingival tissues

\begin{tabular}{ccrrrr}
\hline \multicolumn{1}{c}{ Groups } & $n$ & Mean & $\begin{array}{r}\text { Standard } \\
\text { deviation }\end{array}$ & Minimum & Maximum \\
\hline MVD KCOT & 20 & 30.10 & 10.67 & 11.50 & 47.49 \\
DC & 20 & 12.17 & 5.37 & 5.79 & 11.61 \\
GG & 10 & 8.70 & 7.69 & 8.63 & 16.75 \\
LOXL4 KCOT & 20 & 37.97 & 7.71 & 15.39 & 47.38 \\
DC & 20 & 15.58 & 3.67 & 6.36 & 23.53 \\
GG & 10 & 11.32 & 5.58 & 3.25 & 14.83 \\
\hline
\end{tabular}

DC, dentigerous cysts; GG, gingival primary stromal fibroblast cells; KCOT, keratocystic odontogenic tumors; LOXL4, Iysyl oxidase-like 4; MVD, microvessel density.

MVD was calculated as number of microvessels $\left(1 \times 10^{6} \mu \mathrm{m}^{-2}\right)$. 


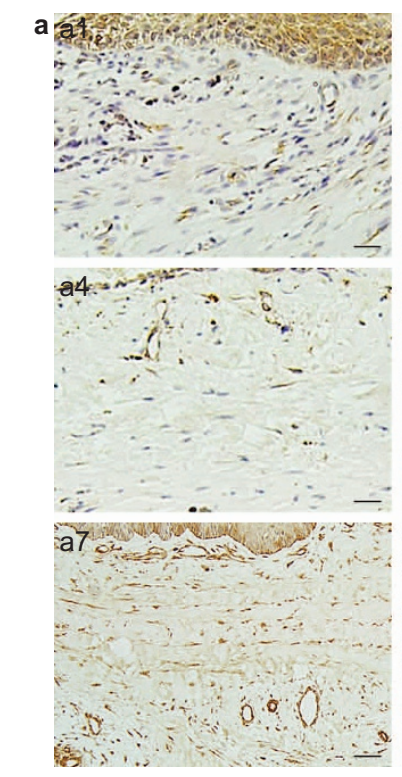

b
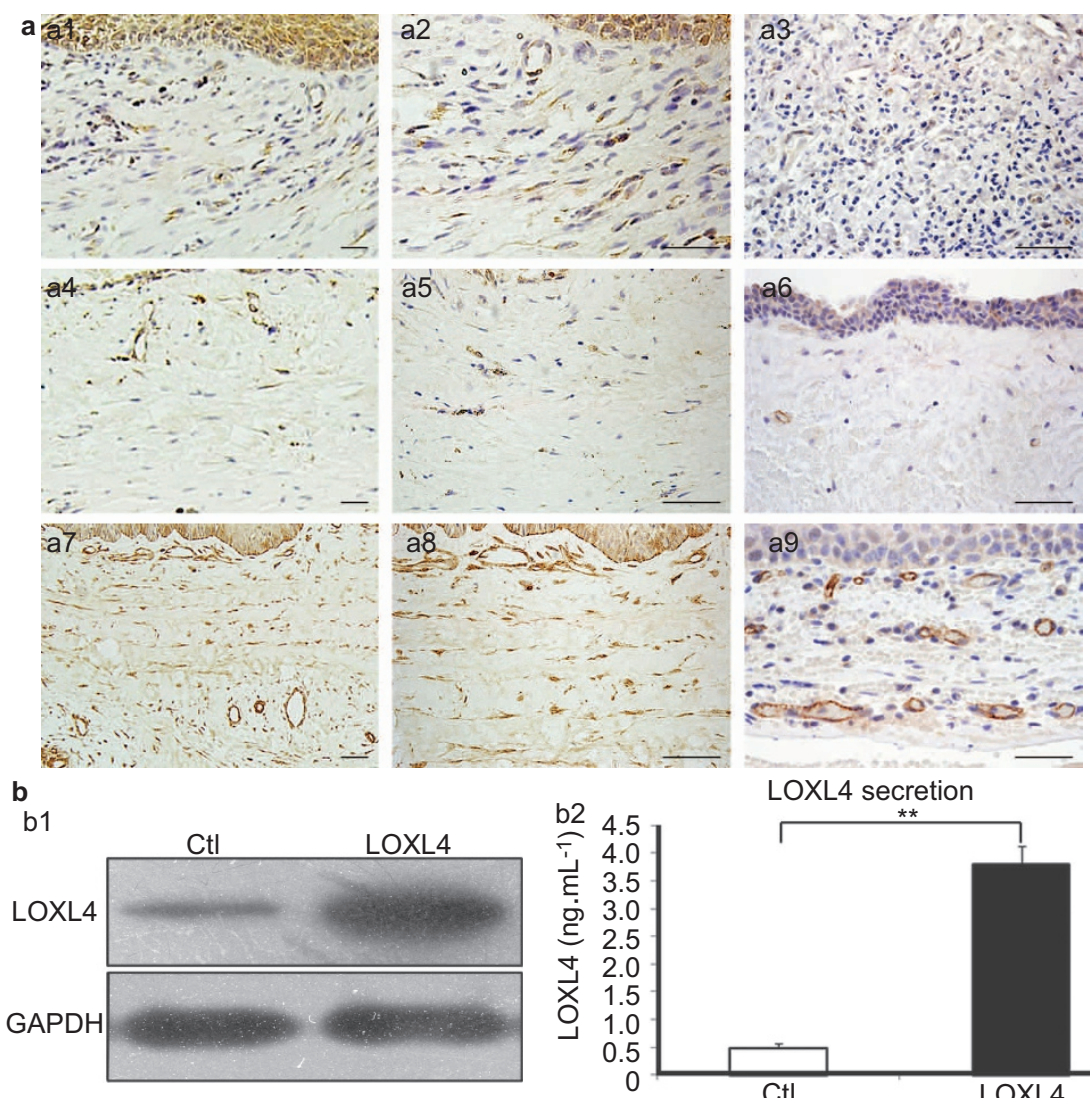

LOXL4 secretion

b3
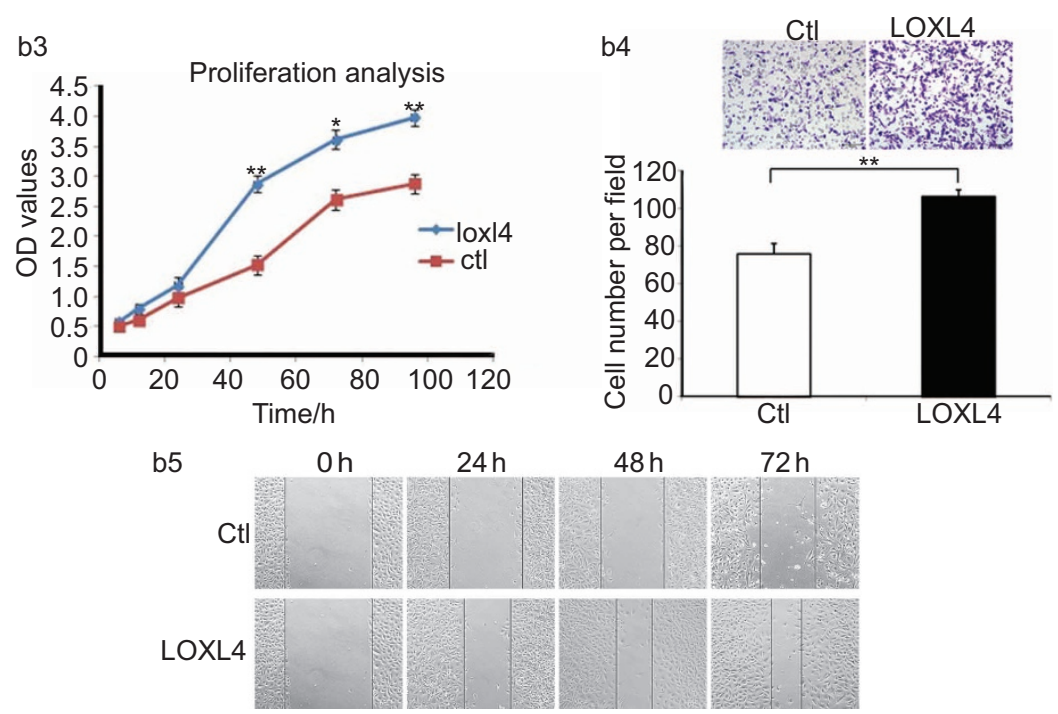

$48 \mathrm{~h}$

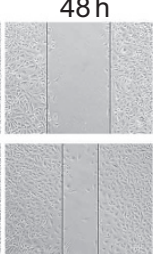

$72 \mathrm{~h}$

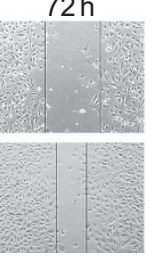

Figure 4 Effects of LOXL4 on angiogenesis. (a) LOXL4 expression and microvessel density in DC, GG and KCOT stroma. Immunohistochemical analysis of LOXL4 (a1, a2, a4, a5). Few LOXL4-positive fibroblasts were observed in GG (a1, a2) or DC (a4, a5). (a7, a8) Most of the KCOT stromal fibroblasts were strongly LOXL4positive. (a3, a6) Few CD105-positive microvessels were observed adjacent to the epithelium of normal GG (a3) and dentigerous cysts (a6). A high density of strongly CD105-positive microvessels was found in the stroma immediately beneath the epithelium lining KCOT (a9). The intensity of LOXL4 protein expression was consistent with the density of CD105-positive microvessels in GG (a3), DC (a6) and KCOT (a9). Scale bar=100 $\mu \mathrm{m}$. (b) Effects of LOXL4 on the proliferation and migration of HUVECs. (b1, b2) There was a significant increase in the expression of LOXL4 both in intracellular and extracellular of HUVECs transfected with LOXL4 than transfected with empty plasmid. (b3) Transient LOXL4 transfection increased cell growth, cell proliferation was measured with Cell Counting Kit-8 at 6, 12, 24, 72 and 96 h. (b4) HUVECs migration ability was assessed by scratching assay with or without LOXL4 transfection. Representative images are shown (magnification: $\times 100$ ). (b5) HUVECs migration ability was also tested by Boyden Chamber assay with or without LOXL4 transfection. Representative images are shown (magnification: $\times 200$ ). Each treatment was triplicate and six fields were randomly chosen from each well for counting mean number of migrated cells. Data are presented as mean \pm s.e.m., $* P<0.05,{ }^{*} P<0.01$, Student's $t$-test. DC, dentigerous cysts; ELISA, enzyme-linked immunosorbent assay; GG, gingival primary stromal fibroblast cells; HUVEC, human umbilical vein endothelial cell; KCOT, keratocystic odontogenic tumors; LOXL4, lysyl oxidase-like 4; OD, optical density; Ctl, control. 


\section{Effects of LOXL4 on HUVEC proliferation study}

HUVEC proliferation study was used to evaluate the effect of LOXL4 in vitro. Anti-LOXL4 antibodies were used to detect exogenous LOXL4 protein, respectively. LOXL4 protein expression (Figure 4b1 and 4b2) was higher in HUVECs transfected with LOXL4 than in control groups (transfected with empty plasmid). Transient overexpression of LOXL4 significantly induced cell proliferation in comparison to control cells (Figure 4b3).

\section{Effects of LOXL4 on HUVEC migration study}

HUVEC migration study was assessed by Transwell assay. As shown in Figure $4 \mathrm{~b} 4$, the number of cells that crossed the membrane in the LOXL4 overexpression groups was significantly higher than in the control groups.

Scratching assays were also performed to determine whether transient LOXL4 expression would also affect the endothelial migration. Figure $4 \mathrm{~b} 5$ indicated that LOXL4 could promote HUVECs' migration.

\section{DISCUSSION}

In 1956, Philipsen ${ }^{24}$ first described a cyst of the jaw lined by keratinizing epithelium, which was termed an odontogenic keratocyst. Based on its unique clinicopathological features, such as aggressive growth within the jaws, tendency to invade surrounding anatomical structures and occasional malignant alteration, ${ }^{25}$ the World Health Organization reclassified odontogenic keratocyst as KCOT in $2005{ }^{26}$ A considerable number of previous studies attributed the highly neoplastic nature of KCOT to the epithelium. ${ }^{427-29}$ However, no persuasive evidence indicates that the epithelium plays a significant role in KCOT. Increasing evidence supports the argument that tumor microenvironment plays a major role in all phases of tumorigenesis, including initiation, progression, maintenance and metastasis, and may also influence therapeutic outcome. In 1975, Browne ${ }^{5}$ first proposed that the connective tissue wall plays a significant role in the pathogenesis of KCOT. Recently, differences in angiogenesis, collagen fibers and osteoclastogenic ability have been identified in the stroma of KCOT and control tissues. ${ }^{6-11}$

In 2011, Gadbail et al. ${ }^{8}$ found that the MVD and the Ki-67 labeling index were significantly higher in the stroma of KCOT than DC and normal oral mucosa, with a significant positive correlation between the Ki-67 and MVD. ${ }^{8}$ This suggests that angiogenesis is associated with the locally aggressive biological behavior of KCOT.

Collagen is a major component of the extracellular matrix (ECM). In a pathological setting, collagen contributes to diameter and crosssectional profile of individual fibers. Hirshberg et al. ${ }^{30}$ has shown that the polarization color pattern of collagen fibers in the connective tissue stroma of KCOT differs from DC. Zhang et al. ${ }^{9}$ reported that the stromal collagen fibers of KCOT were different from those of subcutaneous epidermoid cysts, but similar to those of unicystic ameloblastoma using picrosirius red staining. ${ }^{9}$ These findings lend further support to the hypothesis that KCOT should be regarded as a cystic neoplasm rather than a cyst. ${ }^{26,30-31}$

When osteoclast precursor Raw264.7 cells are cocultured with fibroblasts from KCOT fibrous capsule and gingival mucosa, a significantly higher number of osteoclast-like cells were observed in the KCOT coculture system than in the gingival coculture system. ${ }^{11}$ This suggests that KCOT fibroblasts may be involved in the bone resorption process by interacting with osteoclast precursors and enhances osteoclastogenesis.

However, the mechanisms leading to differences in KCOT stroma remain elusive. To investigate how mesenchymal cells affect the progression of KCOT, we compared the results of RNA-Seq, which compared KCOT stromal fibroblasts with GG stromal fibroblasts, and a microarray analysis which compared KCOT stromal fibroblasts with DC stromal fibroblasts. We filtered the data to identify differentially expressed genes that may play a role in the progression of KCOT, particularly angiogenesis, collagen remodeling and osteoclastogenesis (Table 2 and Figure 2). Based on this analysis, we focused on LOXL4, which encodes a secreted protein that may affect neighboring tumor cells.

LOXL4 was first shown to function as a copper-dependent amine oxidase which catalyzes lysine-derived crosslinks in ECM proteins, and increased expression of LOXL4 has been reported in various of tumors. ${ }^{32}$ We demonstrated that KCOT stromal cells express higher intracellular and extracellular levels of LOXL4 protein than DC or GG stromal cells (Figure 3), indicating that LOXL4 may play a more important role in KCOT stromal cells than in DC or GG stromal cells. Two other LOX family members (LOX and LOXL2), which have similar sequences and structures to LOXL4, are known to promote angiogenesis, ${ }^{22-23}$ leading to the hypothesis that LOXL4 may also participate in angiogenesis. In this study, the mean number of microvessels was significantly higher in KCOT tissues than DC or GG tissues, which corresponds to other reports. ${ }^{8,33-34}$ Transient LOXL4 overexpression in HUVECs confirmed LOXL4-enhanced HUVEC proliferation and migration in vitro (Figure $4 \mathrm{~b} 3,4 \mathrm{~b} 4$ and $4 \mathrm{~b} 5$ ). We also found that MVD was significantly and positively correlated with LOXL4 expression in the stroma of KCOT, DC and GG. This suggests that increased expression and secretion of LOXL4 by stromal cells is related to tumor angiogenesis in KCOT, which in turn may facilitate the locally aggressive biological behavior of this tumor type. ${ }^{8}$

Other candidate genes may play roles in KCOT progression by altering the tumor microenvironment. TCTA may be required for cellular fusion during osteoclastogenesis, and polyclonal antibodies against TCTA could inhibit both human osteoclastogenesis from monocytes and fusion of mature osteoclasts. ${ }^{35}$ This suggests that the high levels of TCTA expression in KCOT stroma may favor osteoclastogenesis. LARP6 could regulate the coordinated translation of type I collagen alpha- 1 and alpha- 2 mRNAs, CO1A1 and CO1A2, and stabilize the mRNAs through high-affinity binding of a stem-loop structure in their 5' UTR. The high expression of LARP6 in KCOT stroma may play a role in the development of tissue fibrosis by stabilizing collagen mRNAs. ${ }^{36-37}$ RGS12 may play a role in tumorigenesis by increasing the GTPase activity of G-protein alpha subunits, thereby driving them into their inactive GDP-bound form to inhibit signal transduction. ${ }^{38}$ Aberrant RGS aberrant plays a pivotal role in vascular maturation and vessel remodeling. ${ }^{39}$ Moreover, RGS12 is essential for the terminal differentiation of osteoclasts induced by RANKL. ${ }^{40}$

In summary, our data demonstrate that abnormal expression of these novel genes in KCOT stromal cells may affect the tumor microenvironment by mediating angiogenesis, collagen remodeling and osteoclastogenesis. These findings support the hypothesis that the stroma of KCOT plays an important role in the neoplastic behavior of this tumor type, as well as providing structural support for the cyst wall. Due to the limited number of specimens used, further studies using other sophisticated methods and larger numbers of patient samples are required to clarify the role of these genes, particularly LOXL4, in KCOT progression. The identification of changes in LOXL4, TCTA, LARP6 and RGS12 expression in KCOT stroma and their potential function in angiogenesis, collagen remodeling and osteoclastogenesis will result in interest in these genes as targets for various KCOT treatment strategies. 


\section{ACKNOWLEDGEMENTS}

This work was supported by the National Natural Science Foundation of China (grant nos. 81030018, 30872900 and 30901680) and the Doctoral Fund of Ministry of Education of China (grant no. 20120001110043). The funders had no role in study design, data collection and analysis, decision to publish or preparation of the manuscript.

1 Mendes RA, Carvalho JF, van der Waal I. Biological pathways involved in the aggressive behavior of the keratocystic odontogenic tumor and possible implications for molecular oriented treatment-an overview. Oral Oncol 2010; 46(1): 19-24.

2 Figueroa A, Correnti M, Avila M et al. Keratocystic odontogenic tumor associated with nevoid basal cell carcinoma syndrome: similar behavior to sporadic type? Otolaryngol Head Neck Surg 2010; 142(2): 179-183.

3 Shear MSP. Cysts of the oral and maxillofacial regions. Oxford: Blackwell Munksgaard, 2007.

4 Pan S, Dong Q, Li TJ et al. Mechanisms of inactivation of PTCH1 gene in nevoid basal cell carcinoma syndrome: modification of the two-hit hypothesis. Clin Cancer Res 2010; 16(2): 442-450.

5 Browne RM. The pathogenesis of odontogenic cysts: a review. J Oral Pathol 1975; 4(1): 31-46.

6 Bhowmick NA, Moses HL. Tumour stroma interactions. Curr Opin Genet Dev 2005; 15(1): 97-101.

7 Park CC, Bissell MJ, Barcellos-Hoff MH. The influence of the microenvironment on the malignant phenotype. Mol Med Today 2000; 6(8): 324-329.

8 Gadbail AR, Hande A, Chaudhary M et al. Gondivkar. Tumor angiogenesis in keratocystic odontogenic tumor assessed by using CD-105 antigen. J Oral Pathol Med 2011; 40(3): 263-269.

9 Zhang JY, Dong Q, Li TJ. Differences in collagen fibres in the capsule walls of parakeratinized and orthokeratinized odontogenic cysts. Int J Oral Maxillofac Surg 2011; 40(11): 1296-1300.

$10 \mathrm{Vij}$ R, Vij H, Rao NN. Evaluation of collagen in connective tissue walls of odontogenic cysts-a histochemical study. J Oral Pathol Med 2011; 40(3): 257-262.

11 Wang HC, Li TJ. The growth and osteoclastogenic effects of fibroblasts isolated from keratocystic odontogenic tumor. Oral Dis 2013; 19(2): 162-168.

12 Tuch BB, Laborde RR, Xu X et al. Tumor transcriptome sequencing reveals allelic expression imbalances associated with copy number alterations. PLOS ONE 2010; 5(2): 9317.

13 Mortazavi A, Williams BA, McCue $\mathrm{K}$ et al. Mapping and quantifying mammalian transcriptomes by RNA-Seq. Nat Methods 2008; 5(7): 621-628.

14 Wang ET, Sandberg R, Luo $S$ et al. Alternative isoform regulation in human tissue transcriptomes. Nature 2008; 456(7221): 470-476.

$15 \mathrm{Li} \mathrm{H}$, Handsaker B, Wysoker A et al. The sequence alignment/map format and SAMtools. Bioinformatics 2009; 25(16): 2078-2079.

16 Levin JZ, Berger MF, Adiconis X et al. Targeted next-generation sequencing of a cancer transcriptome enhances detection of sequence variants and novel fusion transcripts. Genome Biol 2009; 10(10): R115.1-R115.8.

17 Wang Z, Gerstein M, Snyder M. RNA-Seq: a revolutionary tool for transcriptomics. Nat Rev Genet 2009; 10(1): 57-63.

18 Weise JB, Csiszar K, Gottschlich S et al. Vaccination strategy to target lysyl oxidaselike 4 in dendritic cell based immunotherapy for head and neck cancer. Int J Oncol 2008; 32(2): 317-322.

19 Weise JB, Csiszar K, Gottschlich S et al. LOXL4 is a selectively expressed candidate diagnostic antigen in head and neck cancer. Int J Oncol 2008; 44(9): 1323-1331.

20 Jaeger TM, Weidner N, Chew $\mathrm{K}$ et al. Tumor angiogenesis correlates with lymph node metastases in invasive bladder cancer. J Urol 1995; 154(1): 69-71.
21 Wallerman O, Motallebipour $\mathrm{M}$, Enroth $\mathrm{S}$ et al. Molecular interactions between HNF4a, FOXA2 and GABP identified at regulatory DNA elements through ChIPsequencing. Nucleic Acids Res 2009; 37(22): 7498-7508.

22 Shih YH, Chang KW, Chen MY et al. Lysyl oxidase and enhancement of cell proliferation and angiogenesis in oral squamous cell carcinoma. Head Neck 2012; 35(2): 250-256.

23 Bignon M, Pichol-Thievend C, Hardouin J et al. Lysyl oxidase-like protein-2 regulates sprouting angiogenesis and type IV collagen assembly in the endothelial basement membrane. Blood 2011; 118(14): 3979-3989.

24 Philipsen HP. Om keratocystedr (kolesteratomer) i and kaeberne. Tandlaegebladet 1956; 60: 963-971.

25 Jurisic M, Andric M, dos Santos JN et al. Clinical, diagnostic and therapeutic features of keratocystic odontogenic tumors: a review. J BUON 2012; 17(2): 237-244.

26 Barnes L, Eveson J, Reichart P et al. World Health Organization classification of tumours: pathology and genetics of head and neck tumours. Ear Nose Throat $J$ 2006; 85(2): 74

27 Tsuneki M, Cheng J, Maruyama S et al. Perlecan-rich epithelial linings as a background of proliferative potentials of keratocystic odontogenic tumor. J Oral Pathol Med 2008; 37(5): 287-293.

28 Pan S, Li TJ. PTCH1 mutations in odontogenic keratocysts: are they related to epithelial cell proliferation? Oral Oncol 2009; 45(10): 861-865.

29 Mendes RA, Carvalho JF, van der Waal I. A comparative immunohistochemical analysis of COX-2, p53, and Ki-67 expression in keratocystic odontogenic tumors. Oral Surg Oral Med Oral Pathol Oral Radiol Endod 2011; 111(3): 333-339.

30 Hirshberg A, Sherman S, Buchner A et al. Collagen fibres in the wall of odontogenic keratocysts: a study with picrosirius red polarization microscopy. J Oral Pathol Med 1999; 28(9): 410-412.

31 Shear M. The aggressive nature of the odontogenic keratocyst: is it a benign cystic neoplasm? Part 3. Immunocytochemistry of cytokeratin and other epithelial cell markers. Oral Oncol 2002; 38(5): 407-415.

32 Scola N, Gorogh T. LOXL4 as a selective molecular marker in primary and metastatic head/neck carcinoma. Anticancer Res 2010; 30(11): 4567-4571.

33 el-Labban NG, Aghabeigi B. A comparative stereologic and ultrastructural study of blood vessels in odontogenic keratocysts and dentigerous cysts. J Oral Pathol Med 1990; 19(10): 442-446.

34 Alaeddini M, Salah S, Dehghan F et al. Comparison of angiogenesis in keratocystic odontogenic tumours, dentigerous cysts and ameloblastomas. Oral Dis 2009; 15(6): 422-427.

35 Kotake S, Nanke Y, Kawamoto $M$ et al. T-cell leukemia translocation-associated gene (TCTA) protein is required for human osteoclastogenesis. Bone 2009; 45(4): 627 639.

36 Manojlovic Z, Stefanovic. A novel role of RNA helicase A in regulation of translation of type I collagen mRNAs. RNA 2012; 18(2): 321-324.

37 Challa AA, Stefanovic B. A novel role of vimentin filaments: binding and stabilization of collagen mRNAs. Mol Cell Biol 2011; 31(18): 3773-3789.

38 Wang J, Lippman SM, Lee JJ et al. Genetic variations in regulator of G-protein signaling genes as susceptibility loci for second primary tumor/recurrence in head and neck squamous cell carcinom. Carcinogenesis 2010; 31(10): 1755-1761.

39 Manzur M, Hamzah J, Ganss R. Modulation of G protein signaling normalizes tumor vessels. Cancer Res 2009; 69(2): 396-399.

40 Yang S, Li YP. RGS12 is essential for RANKL-evoked signaling for terminal differentiation of osteoclasts in vitro. J Bone Miner Res 2007; 22(1): 45-54.

This work is licensed under a Creative Commons Attribution-NonCommercial-NoDerivative Works 3.0 Unported License. To view a copy of this license, visit http:// creativecommons.org/licenses/by-nc-nd/3.0 